\title{
The Next-Hop Node Selection Based GPSR in Vehicular Ad Hoc Networks
}

\author{
Zhaoyuan Cui1,2, Demin Li1,2*, Guanglin Zhang1,2, Chang Guo',2, Yong Sheng1,2 \\ ${ }^{1}$ College of Information Science and Technology, Donghua University, Shanghai, China \\ ${ }^{2}$ Engineering Research of Center of Digitized Textile and Apparel Technology, Ministry of Education, Shanghai, \\ China \\ Email: zhaoyuancui@mail.dhu.edu.cn,"deminli@dhu.edu.cn,glzhang@dhu.edu.cn, \\ guochang@mail.dhu.edu.cn,yongshengcao@mail.dhu.edu.cn
}

Received 20 June 2016; accepted 12 August 2016; published 15 August 2016

Copyright (C) 2016 by authors and Scientific Research Publishing Inc.

This work is licensed under the Creative Commons Attribution International License (CC BY). http://creativecommons.org/licenses/by/4.0/

(c) () Open Access

\begin{abstract}
Vehicular Ad hoc Networks (VANETs) which is a special form of Mobile Ad hoc Networks (MANETs) has promising application prospects in the future. Due to the rapid changing of topology structure, how to find a route which can guarantee Quality of Service (QoS) is an important issue in VANETs. This paper presents an improved Greedy Perimeter Stateless Routing (GPSR) protocol based on our proposed next-hop node selection mechanism. Firstly, we define the link reliability in two cases which take the movement direction angle between two vehicles into consideration. Then we propose a next-hop node selection mechanism based on a weighted function which consists of link reliability between the sender node and next-hop candidate node, distance between next-hop candidate node and the destination, movement direction angle of next-hop candidate node. At last, an improved GPSR protocol is proposed based on the next-hop node selection mechanism. Simulation results are presented to evaluate the performance of the improved GPSR protocol, which shows that the performance including packet delivery ratio and average end-to-end delay of the proposed protocol is better in some situations.
\end{abstract}

\section{Keywords}

VANETs, GPSR, Next-Hop Node Selection, Link Reliability, Weighted Function

\section{Introduction}

In recent years, Vehicular Ad Hoc Networks (VANETs) [1] has achieved more and more interest from research-

\footnotetext{
"Corresponding author.
}

How to cite this paper: Cui, Z.Y., Li, D.M., Zhang, G.L., Guo, C. and Sheng, Y. (2016) The Next-Hop Node Selection Based GPSR in Vehicular Ad Hoc Networks. Journal of Computer and Communications, 4, 44-56. 
ers all over the world. VANETs can support applications such as car accident warning and entertainment among vehicles through Vehicle-to-Infrastructure (V2I) and Vehicle-to-Vehicle (V2V) communications. For example, when vehicles are traveling on the road, they can share information with each other through V2V and V2I communications. V2V communication is particularly important because of its inherent characters such as free and independent of infrastructure. V2V scenario is extremely important when there is no infrastructure or RSU along the road. The construction and maintenance of infrastructure or base station cost too much. Besides, when base station fails or there is no RSU along the road, vehicles can share safe and entertainment information through V2V communication.

Next-hop node selection is extremely important when the destination node is out of the transmission range of the source node and needs multi-hop to deliver data packets. The selection of next-hop node may affect the QoS such as end-to-end delay and data delivery ratio of a route. Next-hop node selection mechanism is affected by many factors such as link reliability [2], the distance and movement direction angle between a node's next-hop candidate node and the destination node. The high mobility of vehicles in VANETs imposes challenge problems on the analysis of link reliability. The route that is established between a vehicle and another vehicle may be invalid when a vehicle is out of the transmission range of another vehicle.

GPSR [3] protocol is a typical position-based routing protocol that relies on geographic position information. In GPSR protocol, greedy forwarding is used to deliver data packets, that is, a sender node will choose its neighboring node which is closest to the destination as the next-hop node. When the greedy forwarding fails, perimeter forwarding will be used. With the greedy forwarding mechanism, GPSR protocol minimizes the hop number from the source to the destination, however, the transmission quality of the wireless link is ignored.

The improvement of GPSR routing protocol has been studied a lot by many researchers. Rao et al. [4] present a protocol called GPSR-L which is an improved version of GPSR protocol that takes link lifetime into consideration to select the next hop forwarding node. However, authors only consider the situations that vehicles move in same direction and in opposite direction when calculating link lifetime. Authors also assume that vehicle velocity is a constant when calculating link lifetime, however, vehicle velocity should be considered as random variable. Eiza et al. [5] propose a reliable routing protocol known as AODV-R which takes link reliability metric into consideration to improve the performance of conventional AODV protocol. Shelly et al. [6] propose a reliability based GPSR protocol which ensures that links with reliability factor greater than a given threshold alone are selected, when constructing a route from source to destination. Authors in [5] [6] only consider the situation that vehicles move in same direction and in opposite direction when calculating the link reliability, they also don't take other important metrics such as distance and movement direction angles between next-hop candidate node and destination into consideration to select the next-hop node.

This paper focuses on the analysis of an improved GPSR protocol based on a novel next-hop node selection mechanism in VANETs. Our major contributions are listed as follows:

- We propose a next-hop relay node selection mechanism based on a novel weighted function which consists of link reliability between a sender node and its next-hop candidate node, distance and movement direction angle between the next-hop candidate node and the destination node.

- An improved GPSR protocol is proposed based on our proposed next-hop node selection mechanism.

The rest of this paper is organized as follows. The problem is formulated in Section 2. Section 3 presents our proposed novel next-hop node selection mechanism in the V2V scenario. Section 4 enhances the conventional GPSR protocol based on our proposed next-hop node selection mechanism. Section 5 evaluates the performance of the improved GPSR protocol using NS-2 simulator. Conclusion and future work are presented in Section 6.

\section{Problem Formulation}

As shown in Figure 1, vehicle $S$ is the source node, vehicle $D$ is the destination node. Because node $D$ is out of the transmission range of node $S$, so they need multi-hop to communicate with each other. Vehicle $r_{1}, r_{2}, r_{3}$ are relay Nodes.

In our model, we consider the V2V scenario which has no infrastructure or road side unit along the road. Any valuable information can be collected from sensors on a vehicle. The information can be sent to neighboring vehicles. The transmission range of every vehicle is equal, denoted by $R$. The communication link between two vehicles depend only on the distance between them, in other words, as long as one vehicle is in the transmission range of another vehicle, they can communicate with each other. We assume that vehicles are equipped with GPS and digital road maps, that is, every vehicle can obtain its current location and velocity information. 
The implement scenario of our proposed mechanism is shown in Figure 2, the black node $S$ is the sender node, a sender node could be a source node or a relay node. The red node $D$ is the destination node. To reduce number of hops, we assume that the sender vehicle will transmit data in the direction of the line between the sender node and the destination, that is, the nodes in section 1 (S1) are in the direction of the line between the sender node and the destination, similarly, the nodes in section 2 (S2) are not. As shown in Figure 2, nodes in the right semi-circle of the transmission range of the sender node will be considered for packet forwarding. We define all the vehicles in S1 as the next-hop candidate nodes. We have to choose the next-hop node in the all next-hop candidate nodes.

The key idea of our proposed next-hop node selection mechanism is to formulate a weighted function (WF) which comprehensively considers the effect of several factors such as link reliability between a sender node and it's next-hop candidate node, distance between the next-hop candidate node and the destination, movement direction angle of the next-hop candidate node. Details on the proposed mechanism are described below.

\section{Proposed Weighted Function Based Next-Hop Selection Mechanism}

This section describes our proposed next-hop node selection mechanism in the V2V scenario. At first, we define the link reliability in two cases, then we take link reliability, distance and movement direction angle into consideration to formulate the weighted function of a next-hop candidate node. Each next-hop candidate node

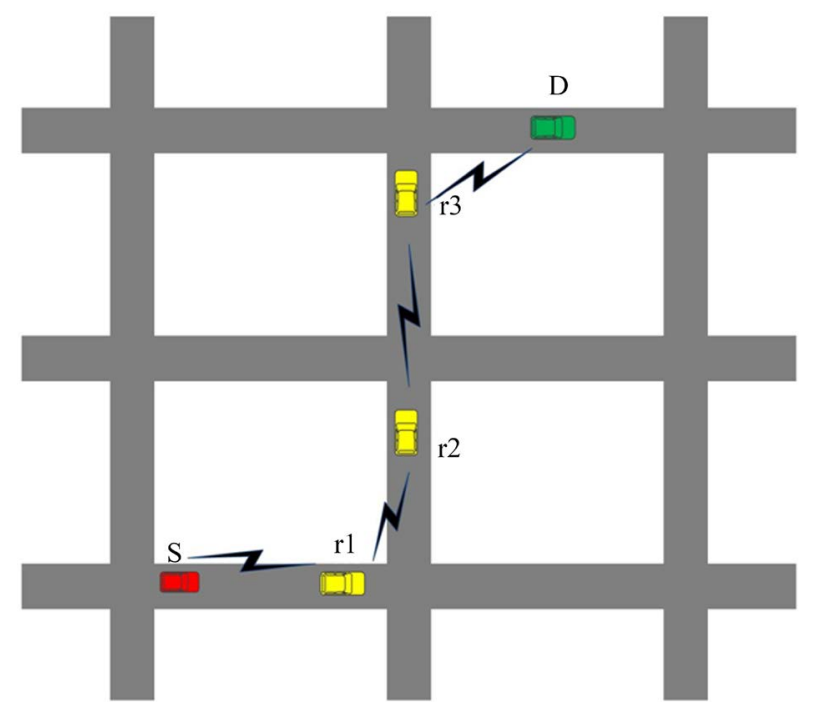

Figure 1. Communication scenario of vehicles traveling on the road.

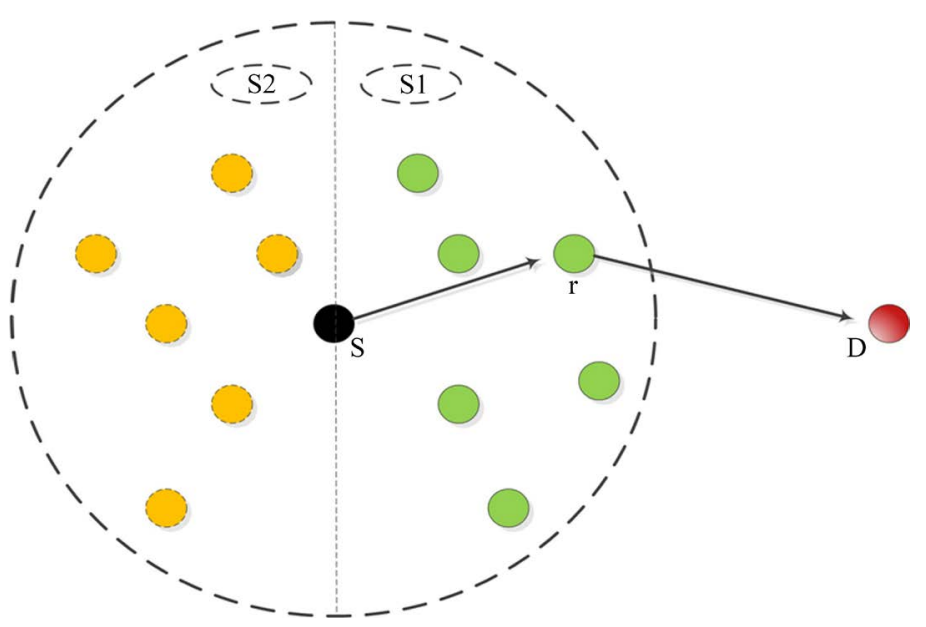

Figure 2. The implement scenario of the proposed mechanism. 
has a weighted function value. The next-hop node selection mechanism is based on the weighted function.

\subsection{The Discussion of the Link Reliability}

In this section, we take the distance, relative velocity and movement direction angle between the sender node and the next-hop candidate node into consideration to analyze the link reliability between the sender node and the next-hop candidate node. Inspired by the work in paper [5] [6], we add movement angle factor when analyzing the link reliability. When the road condition is complex such as crossroad or roundabout lanes, it's necessary to consider movement angle when analyzing link reliability. The link reliability is defined as the probability that an link remain available for a predicted time interval. In this section, we discuss the link reliability in two cases.

1) Case 1: The link reliability when the movement direction angle between the sender node and the next-hop candidate node ranges from 0 to $\pi / 2$.

Now we analyze the link reliability when the velocity direction angle between the sender node and the next-hop candidate node ranges from 0 to $\pi / 2$ As shown in Figure 3, $i$ denotes the sender node, $j$ denotes the next-hop candidate node. $\left(x_{i}, y_{i}\right)$ denotes the position of node $i$, similarly, $\left(x_{i}, y_{i}\right)$ denotes the position of node $j . \overrightarrow{v_{i}}, \overrightarrow{v_{j}}$ denote the velocities of node $i, j$ respectively. $R$ is the transmission range of node $i . D_{i j}$ is the distance between node $i$ and node $j, D_{i j}$ can be calculated by

$$
D_{i j}=\sqrt{\left(y_{j}-y_{i}\right)^{2}+\left(x_{j}-x_{i}\right)^{2}} .
$$

$\theta$ denotes the angle between the movement direction of node $i$ and the movement direction of node $j$ as shown in Figure 4.

We define a coordinate system, node $i$ is the origin, the $x$ axis direction is the positive direction as shown in Figure 5. $\overrightarrow{v_{j x}}=\left(\left|\overrightarrow{v_{j}}\right| \cos \theta\right) \vec{i}, \overrightarrow{v_{j y}}=\left(\left|\overrightarrow{v_{j}}\right| \sin \theta\right) \vec{j}$, where $\theta$ ranges from 0 to $\pi / 2 \cdot d_{r}$ is the relative distance that vehicle $j$ travels in the transmission range of vehicle $i$ as shown in Figure 6. Because link reliability is defined as the probability that a link remains available for a predicted time interval, we need to predict link lifetime before calculating link reliability. by

a) When $\left|\overrightarrow{v_{j x}}\right|>\left|\overrightarrow{v_{i}}\right|$, as shown in Figure 5, the relative velocity between node $i$ and node $j$ can be calculated

$$
\left|\overrightarrow{v_{r}}\right|=\sqrt{\left(\left|\overrightarrow{v_{j x}}\right|-\left|\overrightarrow{v_{i}}\right|\right)^{2}+\left|\overrightarrow{v_{j y}}\right|^{2}}
$$

The relative distance that vehicle $j$ travels in the transmission range of vehicle $i$ is shown in Figure 6. AC is

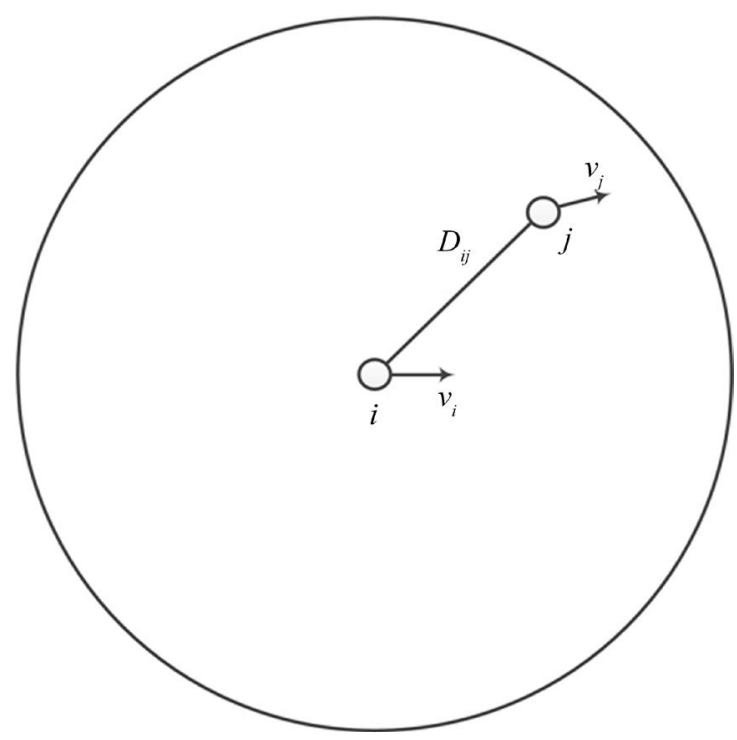

Figure 3. The movement direction angle between node $i$ and node $j$ ranges from 0 to $\pi / 2$. 


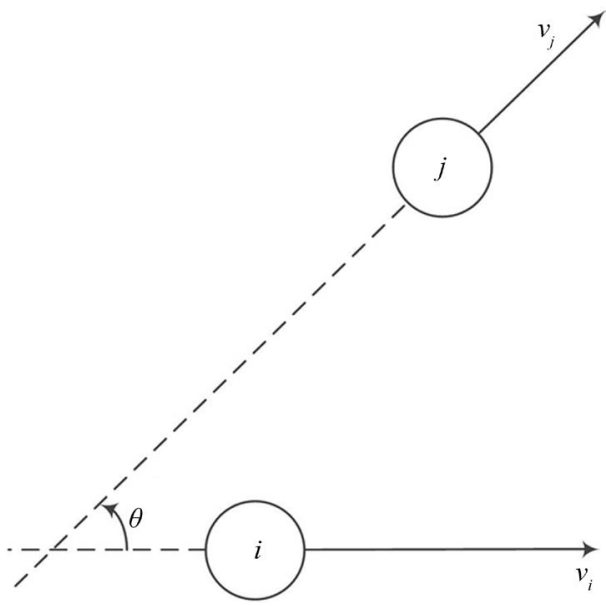

Figure 4. The movement direction angle $\theta$.

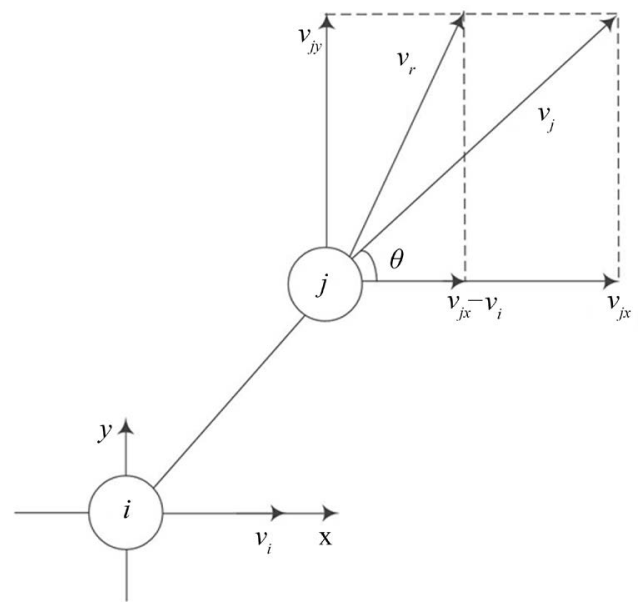

Figure 5. The calculation of relative velocity between node $i$ and node $j$.

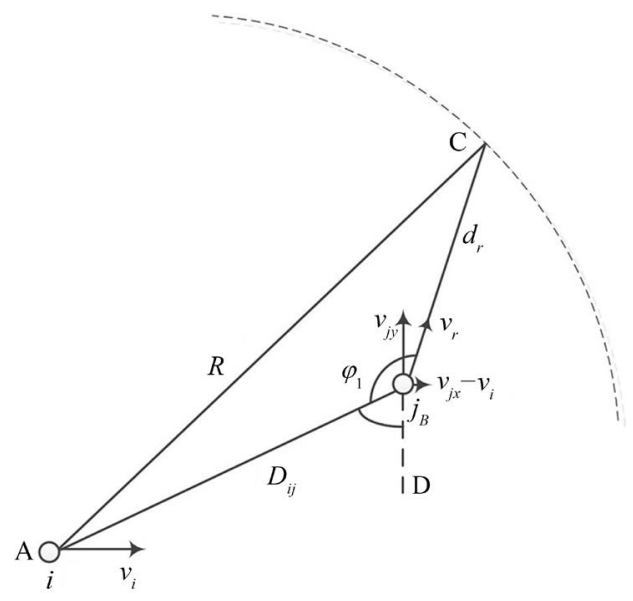

Figure 6. The calculation of relative distance that node $j$ travels in the transmission range of node $i$.

the transmission range $R, A B$ is the distance between node $i$ and node $j, \angle A B C=\varphi_{1}, A D$ is the distance between node $i$ and node $j$. $B C=d_{r}$ is the relative distance which node $j$ travels in the transmission range of node $i$.

The angle $\varphi_{1}$ of the triangle $\triangle A B C$ can be calculated by 


$$
\varphi_{1}=\angle A B C=\pi-\arccos \frac{\left|y_{j}-y_{i}\right|}{D_{i j}}+\arctan \frac{\left|\overrightarrow{v_{j x}}\right|-\left|\overrightarrow{v_{i}}\right|}{\left|\overrightarrow{v_{j y}}\right|}
$$

where $\arccos \frac{\left|y_{j}-y_{i}\right|}{D_{i j}}$ is the angle $\angle A B D, \arctan \frac{\left|\overrightarrow{v_{j x}}\right|-\left|\overrightarrow{v_{i}}\right|}{\left|\overrightarrow{v_{j y}}\right|}$ is the angle between $B C$ and $\overrightarrow{v_{j y}}$.

According to cosine formula $\cos \varphi_{1}=\frac{D_{i j}^{2}+d_{r}^{2}-R^{2}}{2 D_{i j} d_{r}}$, the relative distance $d_{r}$ that vehicle $j$ travels in the transmission range of vehicle $i$ is given by

$$
d_{r}=\sqrt{R^{2}+\left(D_{i j} \cos \varphi_{1}\right)^{2}-D_{i j}^{2}}+D_{i j} \cos \varphi_{1} .
$$

According to Equation (2) and Equation (4), the link lifetime can be calculated by

$$
T_{i j}=\frac{d_{r}}{\left|\overrightarrow{v_{r}}\right|}
$$

where $d_{r}$ is the relative distance which node $j$ travels in the transmission range of node $i .\left|\vec{v}_{r}\right|$ is the relative velocity between node $j$ and node $i$.

We assume that the velocity of vehicles is normal distributed, According to [5], we can get the link reliability as follows:

$$
L R_{i j}= \begin{cases}\int_{t}^{t+T_{i j}} P_{t}(T) \mathrm{d} t & T_{i j}>0 \\ 0 & T_{i j}=0\end{cases}
$$

where $L R_{i j}$ is the link reliability between node $i$ and node $j, T_{i j}$ is the link lifetime between node $i$ and node $j$, $P_{t}(T)$ is the $p d f$ of the communication duration. by

b) When $\left|\overrightarrow{v_{j x}}\right| \leq\left|\overrightarrow{v_{i}}\right|$, as shown in Figure 7, the relative velocity between node $i$ and node $j$ can be calculated

$$
\left|\overrightarrow{v_{r}}\right|=\sqrt{\left(\left|\overrightarrow{v_{i}}\right|-\left|\overrightarrow{v_{j x}}\right|\right)^{2}+\left|\overrightarrow{v_{j y}}\right|^{2}}
$$

As shown in Figure 8, the angle $\varphi_{1}$ of the triangle $\triangle A B C$ can be calculated by

$$
\varphi_{1}=\angle A B C=\pi-\arccos \frac{\left|y_{j}-y_{i}\right|}{D_{i j}}-\arctan \frac{\left|\overrightarrow{v_{i}}\right|-\left|\overrightarrow{v_{j x}}\right|}{\left|\overrightarrow{v_{j y}}\right|} .
$$

Similarly, the link reliability between node $i$ and node $j$ can be calculated by

$$
L R_{i j}= \begin{cases}\int_{t}^{t+T_{i j}} P_{t}(T) \mathrm{d} t & T_{i j}>0 \\ 0 & T_{i j}=0\end{cases}
$$

2) Case 2: The link reliability when the movement direction angle between the sender node and the next-hop candidate node ranges from $\pi / 2$ to $\pi$.

Now we analyze the link reliability when the movement direction angle between the sender node and the next-hop candidate node ranges from $\pi / 2$ to $\pi$ as shown in Figure 9, we can calculate the link reliability with the method in case 1 .

As shown in Figure 10, we can know that $\overrightarrow{v_{j x}}=-\left|\overrightarrow{v_{j}}\right| \cos (\pi-\theta) \vec{i}, \overrightarrow{v_{j y}}=\left|\overrightarrow{v_{j}}\right| \sin (\pi-\theta) \vec{j}$, where $\theta$ ranges from $\pi / 2$ to $\pi$.

Similarly, the relative velocity between node $i$ and node $j$ can be calculated by

$$
\left|\overrightarrow{v_{r}}\right|=\sqrt{\left(\left|\overrightarrow{v_{i}}\right|+\left|\overrightarrow{v_{j x}}\right|\right)^{2}+\left|\overrightarrow{v_{j y}}\right|^{2}} .
$$




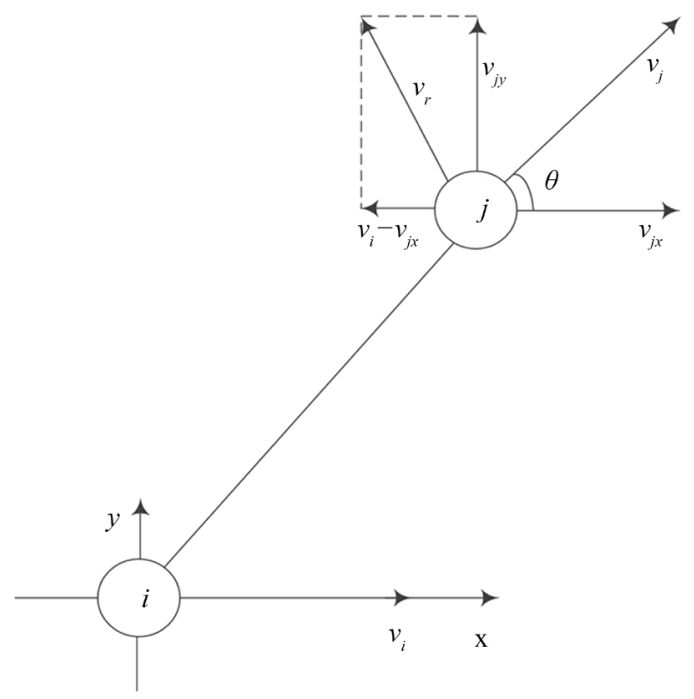

Figure 7. The calculation of relative velocity between node $i$ and node $j$.

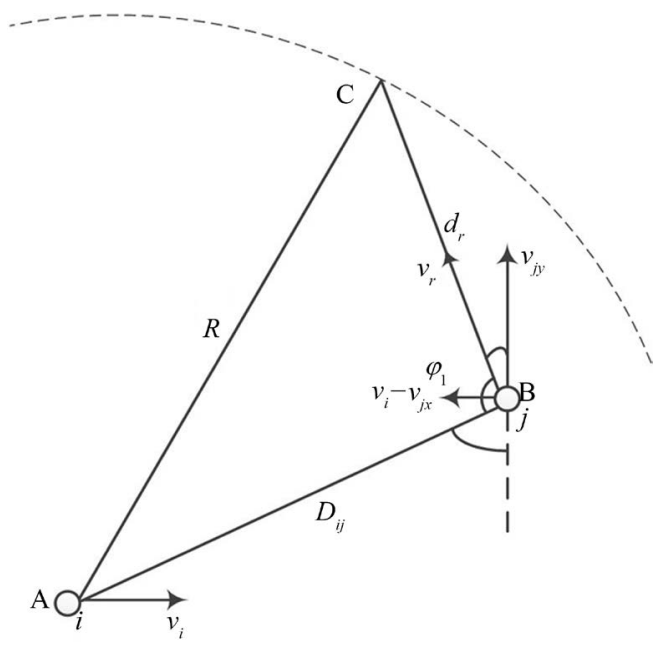

Figure 8. The calculation of relative distance that node $j$ travels in the transmission range of node $i$.

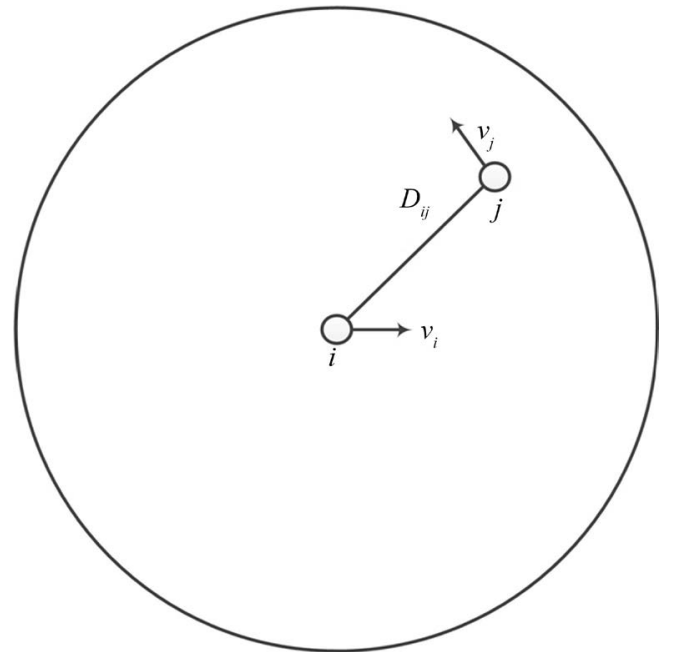

Figure 9. The movement direction angle between node $i$ and node $j$ ranges from $\pi / 2$ to $\pi$. 


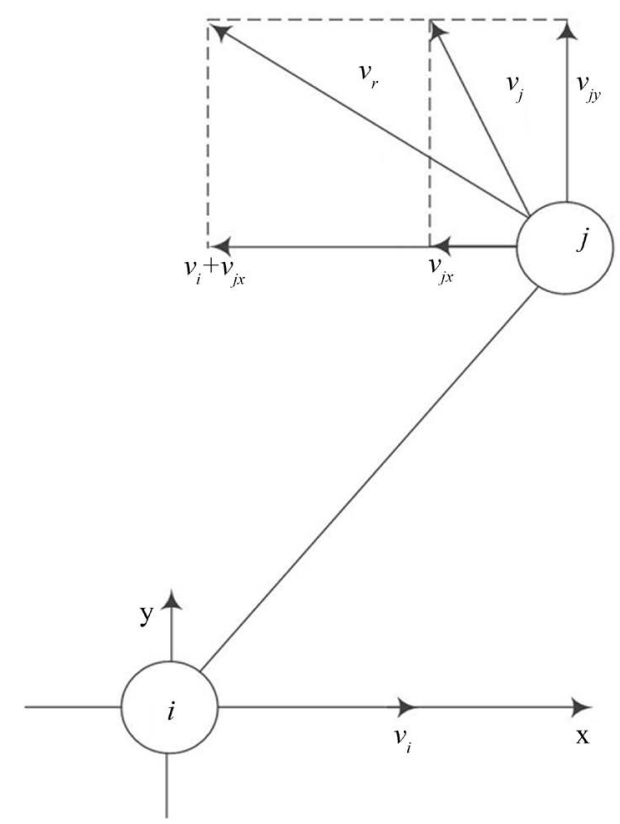

Figure 10. The calculation of relative velocity between node $i$ and node $j$.

So as shown in Figure 11, the angle $\varphi_{2}$ of the triangle $\triangle A B C$ can be calculated by

$$
\varphi_{2}=\angle A B C=\pi-\arccos \frac{\left|y_{j}-y_{i}\right|}{D_{i j}}-\arctan \frac{\left|\overrightarrow{v_{i}}\right|+\left|\overrightarrow{v_{j x}}\right|}{\left|\overrightarrow{v_{j y}}\right|} .
$$

Similarly, we can get the link reliability as follows:

$$
L R_{i j}=\left\{\begin{array}{ll}
\int_{t}^{t+T_{i j}} P_{t}(T) \mathrm{d} t & T_{i j}>0 \\
0 & T_{i j}=0
\end{array} .\right.
$$

In this section, we discussed the link reliability between node $i$ and its next-hop candidate node $j$ in two cases. Then we will analyze the weighted function of next-hop candidate node and our proposed next-hop selection mechanism.

\subsection{The Definition of the Weighted Function (WF) and WF Based Next-Hop Node Selection Mechanism}

In this section, we take the link reliability between the sender node and the next-hop candidate node, distance between the next-hop candidate node and the destination, movement direction angle of the next-hop candidate node into consideration to formulate the weighted function (WF) which is used for the selection of next-hop node. We define WF to ensure both link reliability and hop number. We compare those next-hop candidate vehicles’ WF value and select the vehicle which has the largest WF value as the next-hop relay node.

The weighted function of the next-hop candidate node $j$ is defined as follows:

$$
W F_{i j}=w_{1} L R_{i j}+w_{2} \frac{1}{D_{j d}}+w_{3} \cos \Phi_{j d}
$$

where $w_{1}+w_{2}+w_{3}=1$, $i$ denotes the sender node, $j$ denotes the next-hop candidate node, $d$ denotes the destination node. $D_{j d}$ denotes the distance between the next-hop candidate node $j$ and the destination node $d$. If the locations of $j$ node and the destination node respectively are $\left(x_{j}, y_{j}\right),\left(x_{d}, y_{d}\right)$, then the distance between these two nodes is given b $D_{j d}=\sqrt{\left(x_{d}-x_{j}\right)^{2}+\left(y_{d}-y_{j}\right)^{2}}$. The smallest $D_{j d}$ can minimize the multi-hop number and lead to the smallest end-to-end delay. 
We define $\Phi$ in Figure 12. $\Phi$ is the angle between the movement direction of the next-hop candidate node and the link from the candidate node to the destination node. From the definition of $\Phi$, we can know that $\Phi \in[0, \pi]$ and $\cos \Phi \in[-1,1]$. When the next-hop candidate node is moving forward to the destination, $\cos \Phi=1$. Otherwise, the vehicle is moving opposite the destination, $\cos \Phi=-1$. Obviously, when the value of $\cos \Phi$ is larger, the probability that the link has small hop number is larger for a certain time internal.

We assume that there are $k$ next-hop candidate nodes in the transmission range of the node $i$, so the set of link reliability is $\left\{L R_{i 1}, L R_{i 2}, \cdots, L R_{i k}\right\}$, similarly, the set of $W F$ is $\left\{W F_{i 1}, W F_{i 2}, \cdots, W F_{i k}\right\}$. The next-hop candidate node which has the largest WF will be selected as the next-hop relay node.

The proposed selection mechanism of next-hop node is shown in Algorithm 1.

\section{Improvement of GPSR Protocol Based on Proposed Mechanism}

In this section, we improve the conventional GPSR protocol based on our proposed next-hop node selection mechanism.

\subsection{The Introduction of GPSR Protocol}

GPSR [7] protocol is a typical routing protocol based on geographical position which is suitable for VANETnetwork. In GPSR, every node maintains the information of its one-hop neighboring nodes. Each node participating in routing process selects the next-hop node which is closest to the destination, this procedure is called greedy forwarding. By exchanging periodic HELLO messages among nodes, the locations of neighboring

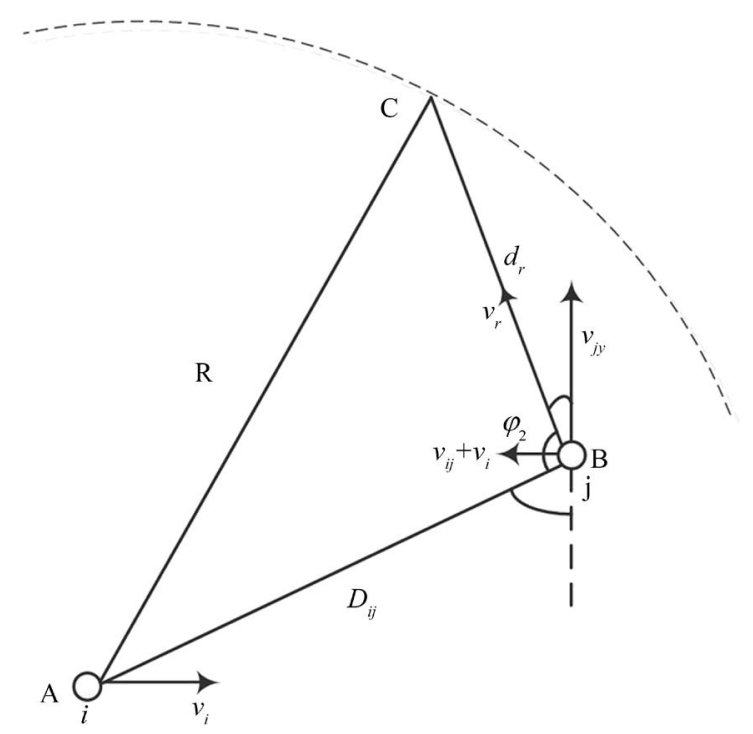

Figure 11. The calculation of relative distance that node $j$ travels in the transmission range of node $i$.

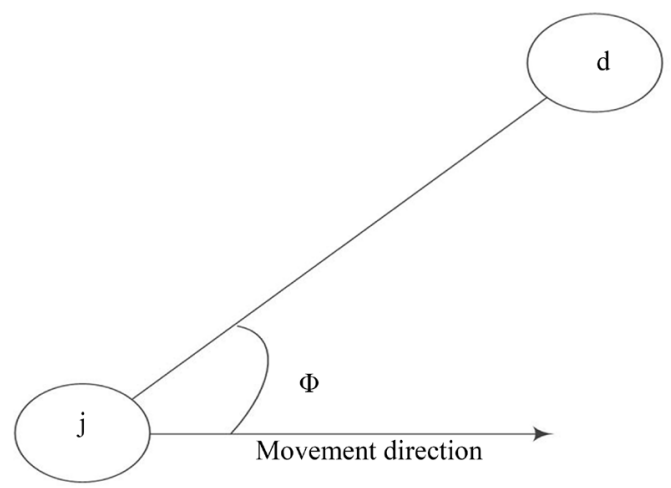

Figure 12. The definition of $\Phi$. 
Algorithm 1. The proposed next-hop node selection mechanism.

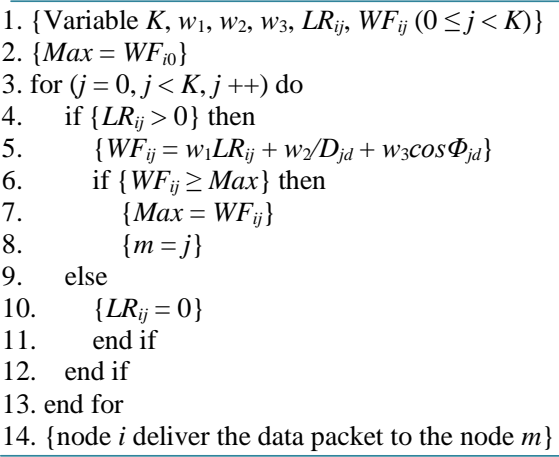

nodesare obtained. When a node receives a HELLO message from its neighbors, it sets the HELLO timer for each of its neighbors. If it does not receive HELLO message from a neighbor before the HELLO timer expires, it assumes that the neighbor has gone out of its transmission range [8].

The greedy forwarding mechanism will always choose the node which is closest to the destination node as the next-hop relay node. Because of high mobility of vehicles, greedy forwarding will lead poor link quality and the cavity phenomenon. To settle these problems which are caused by greedy forwarding, we propose an improved GPSR protocol based on the proposed next-hop node selection mechanism.

\subsection{The Improvement of GPSR Protocol}

In this section we use the proposed next-hop selection mechanism to improve the performance of GPSR protocol (WF-GPSR) to improve the link quality, reduce the cavity phenomenon.

As mentioned above, we comprehensively take the link probability between the sender node and next-hop candidate node, the distance between next-hop candidate node and destination node, the movement direction of next-hop candidate node into consideration to formulate the weighted function (WF) which is used for the selection of next-hop relay node.

$$
W F_{i j}=w_{1} L R_{i j}+w_{2} \frac{1}{D_{j d}}+w_{3} \cos \Phi_{j d} .
$$

To increase the stability of communication route, we choose the next-hop candidate node which has the largest WF as the next-hop node to forwarding data packet in the process of route discovery of GPSR.

The format of "HELLO" package is shown in Figure 13.

We add the WF value of the next-hop candidate node into protocol header. The format of the proposed routing protocol header is shown in Figure 14.

\section{Performance Evaluations}

In this paper, we use NS2.33 which can simulate the process of wireless communication as the simulation platform to evaluate the performance of our proposed protocol WF-GPSR. We have to compare the performance of our proposed protocol WF-GPSR, GPSR-R which is proposed in paper [6], and the conventional GPSR in the same simulation environment. VANETMobisim is used to set up the traffic pattern, including the vehicular velocity distribution. The settings of simulation scenario are described in Table 1.

We set $w_{1}$ as $0.3, w_{2}$ as $0.6, w_{3}$ as 0.1 . Utilizing the system parameters, we evaluate the performance of our proposed protocol WF-GPSR.

In order to show the performance of the WF-GPSR protocol, we compare the packet delivery ratio and the average end-to-end delay of WF-GPSR, GPSR-R, which is proposed in [6], and GPSR protocol. The simulation results of packet delivery ratio are shown in Figure 15. The simulation results of average end-to-end delay are shown in Figure 16.

The results in Figure 15 show that the packet delivery ratio of WF-GPSR is bigger than the packet delivery ratio of GPSR and smaller than the packet delivery ratio of GPSR-R when the vehicle density is big. The packet delivery ratio increases with the increase of vehicle density. When the vehicle density is small, the packet deli- 
very ratio of WF-GPSR is almost the same with GPSR and smaller than GPSR-R. It can be explained as follows: The GPSR-R protocol has more requirements for link reliability, that is, when neighboring nodes' link reliability is too small, the sender node will not deliver the packet to guarantee packet delivery ratio. In WF-GPSR protocol, we consider not only the link reliability but also the distance between the candidate node and the destination and the movement direction of the candidate nodes, so the packet delivery ratio is smaller than GPSR-R and bigger than GPSR.

The simulation results in Figure 16 show that the average end-to-end delay of WF-GPSR protocol is bigger than the GPSR protocol and smaller than the GPSR-R protocol. The average end-to-end delay of these three protocols is no much difference when the vehicle density is very big, however, when the vehicle density is small, the average end-to-end delay of WF-GPSR protocol is bigger than GPSR and smaller than GPSR-R. It can be explained as follows. When the vehicle density is small, the source node or the sender node couldn't find any node as the relay node to deliver data packet when there is link reliability limits in GPSR-R. This mechanism will lead much big delay when the vehicle density is small. In WF-GPSR, we consider the effect of both link reliability and hop number, so the delay of WF-GPSR is bigger than GPSR and smaller than GPSR-R.

The simulation results show that the packet delivery ratio of the WF-GPSR protocol is better than the GPSR protocol when the vehicle density is big, the delay of the WF-GPSR is smaller than the GPSR-R when the vehicle density is small.

\begin{tabular}{|c|l|l|l|}
\hline Node Id & Node Position & $\begin{array}{c}\text { Node } \\
\text { Direction }\end{array}$ & Time stamp \\
\hline
\end{tabular}

Figure 13. The format of HELLO package.

\begin{tabular}{|c|c|c|c|c|c|c|}
\hline Src Id & Dest Id & $\begin{array}{c}\text { Next hop } \\
\text { Id }\end{array}$ & $\begin{array}{c}\text { Dest } \\
\text { Position }\end{array}$ & $\begin{array}{l}\text { Time } \\
\text { stamp }\end{array}$ & WF & Data \\
\hline
\end{tabular}

Figure 14. The format of the WF-GPSR protocol header.

Table 1. Parameter setting in simulations.

\begin{tabular}{cc} 
Item & Value \\
MAC layer & 802.11 \\
Packet size & 512 bytes \\
Channel bit rate & $2 \mathrm{Mbps}$ \\
Transmission range & $250 \mathrm{~m}$ \\
Ad-hoc routing protocol & GPSR and WF-GPSR \\
Simulation area & $5 \times 5 \mathrm{~km}{ }^{2}$ \\
Velocity distribution model & Normal \\
Vehicle maximal speed & $108 \mathrm{kmph}$ \\
Vehicle average speed & $72 \mathrm{kmph}$ \\
Simulation time & $300 \mathrm{~s}$ \\
GPSR beacon interval & $5 \mathrm{~s}$ \\
Vehicle minimal density & $20 \mathrm{veh} / 1000 \mathrm{~m}$ \\
Vehicle maximal density & $50 \mathrm{veh} / 1000 \mathrm{~m}$ \\
CBR packet size & $32 \mathrm{~B}$ \\
CBR send interval & $2 \mathrm{~s}$ \\
\hline
\end{tabular}




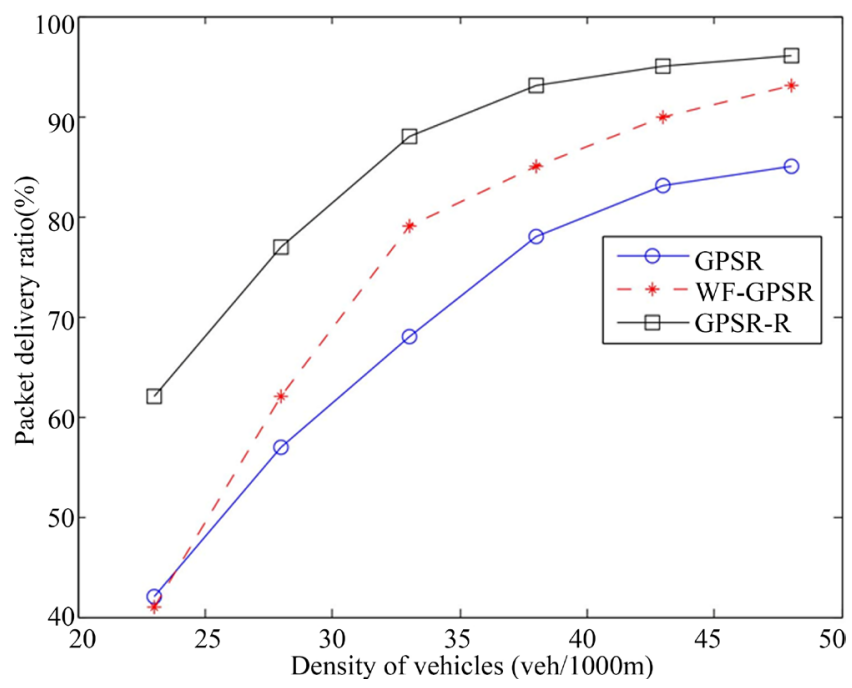

Figure 15. The packet delivery ratio of GPSR, GPSR-R and WF-GPSR with different vehicle densities.

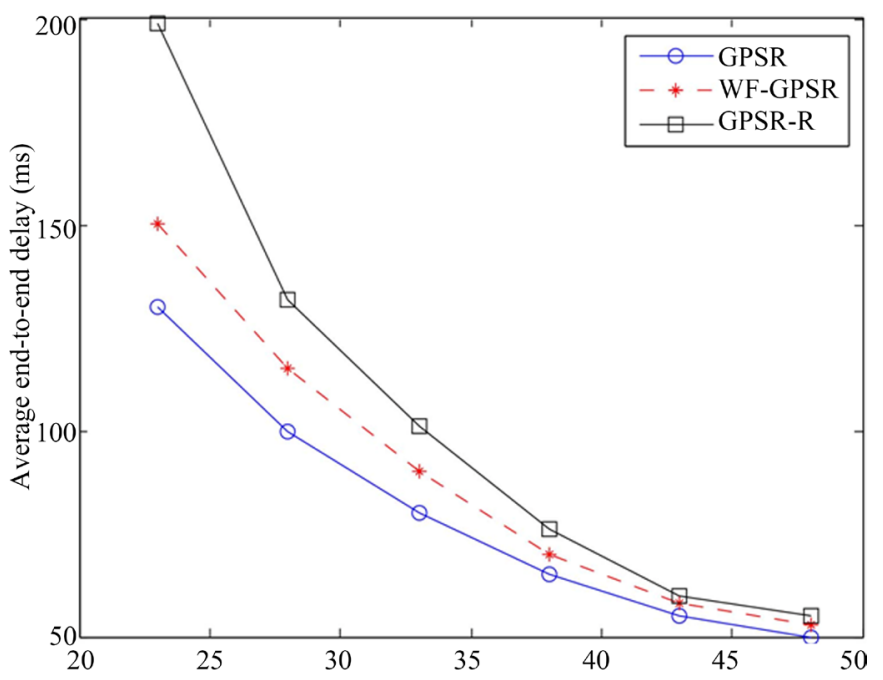

Figure 16. The average end-to-end delay of GPSR, GPSR-R and WF-GPSR with different vehicle densities.

\section{Conclusion}

In this paper, an improved GPSR protocol based on next-hop node selection mechanism in the V2V scenario is studied. The improved protocol which is named as WF-GPSR protocol is proposed based on the analysis of a weight function which consists of link reliability between the sender node and next-hop candidate node, the distance between next-hop candidate node and destination and the movement direction of next-hop candidate node. The simulation results show that the performance of WF-GPSR protocol is better in some situations.

\section{Acknowledgements}

This work is supported by the NSF of China under Grant No. 61301118; the Innovation Program of Shanghai Municipal Education Commission under Grant No. 14YZ130; the International S \& T Cooperation Program of Shanghai Science and Technology Commission under Grant No. 15220710600; and the Fundamental Research Funds for the Central Universities under Grant No. 16D210403.

\section{References}

[1] Shao, C., Leng, S., Zhang, Y., Vinel, A. and Jonsson, M. (2015) Performance Analysis of Connectivity Probability and 
Connectivity-Aware MAC Protocol Design for Platoon-Based VANETs. IEEE Transactions on Vehicular Technology, 64, 5596-5609. http://dx.doi.org/10.1109/TVT.2015.2479942

[2] Eiza, M.H. and Ni, Q. (2012) A Reliability-Based Routing Scheme for Vehicular Ad Hoc Networks (VANETs) on Highways. 2012 IEEE 11th International Conference on Trust, Security and Privacy in Computing and Communications (TrustCom), Liverpool, 25-27 June 2012, 1578-1585

[3] Karp, B. and Kung, H.T. (2000) GPSR: Greedy Perimeter Stateless Routing for Wireless Networks. Proceedings of the ACM/IEEE International Conference on Mobile Computing and Networking (MobiCom), Boston, MA, August 2000, 243-254. http://dx.doi.org/10.1145/345910.345953

[4] Rao, S.A., Pai, M., Boussedjra, M. and Mouzna, J. (2008) GPSR-L: Greedy Perimeter Stateless Routing with Lifetime for VANET. Proceedings of the ITS International Conference on ITS Telecommunications (ITST), Phuket, 24-24 October 2008, 299-304. http://dx.doi.org/10.1109/itst.2008.4740275

[5] Eiza, M.H., Ni, Q., Owens, T. and Min, G. (2013) Investigation of Routing Reliability of Vehicular Ad Hoc Networks. EURASIP Journal on Wireless Communications and Networking, 1, Article 179.

[6] Shelly, S. and Babu, A.V. (2015) Link Reliability Based Greedy Perimeter Stateless Routing for Vehicular Ad Hoc Networks. International Journal of Vehicular Technology, 2015, Article ID: 921414. http://dx.doi.org/10.1155/2015/921414

[7] Hu, T.L., Liwang, M.H., Huang, L.F. and Tang, Y.L. (2015) An enhanced GPSR Routing Protocol Based on the Buffer Length of Nodes for the Congestion Problem in VANETs. 2015 10th International Conference on Computer Science \& Education (ICCSE), Cambridge, 22-24 July 2015, 416-419.

[8] Ning, Z., Jung, Y., Jin, Y. and Kim, K.-C. (2009) Route Optimization for GPSR in VANET. IEEE International Advance Computing Conference (IACC 2009), Patiala, India, 6-7 March 2009, 569-573. http://dx.doi.org/10.1109/iadcc.2009.4809074

\section{Submit or recommend next manuscript to SCIRP and we will provide best service for you:}

Accepting pre-submission inquiries through Email, Facebook, LinkedIn, Twitter, etc.

A wide selection of journals (inclusive of 9 subjects, more than 200 journals)

Providing 24-hour high-quality service

User-friendly online submission system

Fair and swift peer-review system

Efficient typesetting and proofreading procedure

Display of the result of downloads and visits, as well as the number of cited articles

Maximum dissemination of your research work

Submit your manuscript at: http://papersubmission.scirp.org/ 